\title{
Accuracy of Multiplanar MR Images in Determination of Actual Tumour Size in Comparison to the Pelvic Examination of Carcinoma Cervix
}

\author{
Husnaion Zubery ${ }^{1}$, Mahbuba Shirin ${ }^{2}$, Falguni Binte Rahman ${ }^{3}$, Biswajit Bhowmik ${ }^{2}$, \\ Mohammad Ibrahim ${ }^{4}$, Zakia Sultana ${ }^{4}$ \\ ${ }^{1}$ Department of Radiology and Imaging, Rajshahi Medical College Hospital, Rajshahi, Bangladesh \\ ${ }^{2}$ Department of Radiology and Imaging, Bangabandhu Sheikh Mujib Medical University, Dhaka, Bangladesh \\ ${ }^{3}$ Department of Pharmacology and Therapeutics, Rajshahi Medical College, Rajshahi, Bangladesh \\ ${ }^{4}$ Directorate General of Health Services, Dhaka, Bangladesh
}

Email address:

mohammadzubery@gmail.com (H. Zubery)

\section{To cite this article:}

Husnaion Zubery, Mahbuba Shirin, Falguni Binte Rahman, Biswajit Bhowmik, Mohammad Ibrahim, Zakia Sultana. Accuracy of Multiplanar MR Images in Determination of Actual Tumour Size in Comparison to the Pelvic Examination of Carcinoma Cervix. International Journal of Clinical Oncology and Cancer Research. Vol. 6, No. 3, 2021, pp. 136-140. doi: 10.11648/j.ijcocr.20210603.16

Received: July 7, 2021; Accepted: July 24, 2021; Published: August 2, 2021

\begin{abstract}
Accurate staging of patients with cervical carcinoma is crucial for appropriate management planning and tumor size has consistently been a key component \& major determinant when deciding management plan in especially with earlystages as it dictates whether a patient is a candidate for conservative surgery, radical hysterectomy or chemoradiation. Tumor size also provides a prognostic value, as larger tumors predict risk for distant spread of disease and poor prognostic outcomes. The objective of this study was to determine the sensitivity and specificity of Magnetic Resonance Imaging in the measurement of actual tumor size of invasive cervical carcinoma in comparison to pelvic examination taking histopathology as gold standard. This prospective cross sectional study was conducted in the Department of Radiology and Imaging, BSMMU, Dhaka during the period of September 2018 to August 2020. A total of 60 patients were selected purposively and all were undergone pelvic examination clinically by EUA. Then all of them underwent MRI in Department of Radiology and Imaging, BSMMU. Images of uterine cervix, corpus, vagina and parametrium are taken with a prefixed standard protocol and reporting was done by Radiologist. Comparison was done between the MRI and clinical assessment of actual tumour size. Out of 60 patients in this study the mean age was found $47.5 \pm 10.1$ years with range from 22 to 60 years. Positive correlation ( $\mathrm{r}=0.993$; $\mathrm{p}=0.001)$ between histopathological size and MRI size of tumour. Positive correlation $(\mathrm{r}=0.950 ; \mathrm{p}=0.001)$ between histopathological size and FIGO size of tumour. MRI findings more correlates with histopathology than clinically detected tumor size. Based on pathologic findings, accuracy was estimated by the degree of agreement with a difference of $<0.5$ or 1.0 $\mathrm{cm}$ between the measurements of tumor size obtained by pelvic examination and imaging modality. Pelvic examination and imaging modality had an accuracy of $53.3 \%$ and $75 \%$, respectively, with a difference of $<0.5 \mathrm{~cm}$, and an accuracy of $88.3 \%$ and $100 \%$, respectively, with a difference of $0.5-1.0 \mathrm{~cm}$. The study also concluded MRI staging was more concordant with pathological stage compared to the clinical stage. Concordance rate of $95.0 \%$ in MRI and $65.0 \%$ in clinical staging respectively. FIGO staging concurred with histopathology in $39(65.0 \%)$ cases and differed in $21(35.0 \%)$ cases. Magnetic resonance imaging (MRI) is a sensitive and specific modality for accurate determination of tumour size as well as staging of cervical carcinoma in comparison to pelvic examination taking histopathology as gold standard.
\end{abstract}

Keywords: Cervical Carcinoma, MRI, FIGO, Histopathology

\section{Introduction}

Carcinoma of the uterine cervix is the fourth most commonly occurring cancer in women followed by lung, breast and colorectal cancer. Worldwide incidence of carcinoma of the uterine cervix is $3.2 \%$ with the most 
commonly diagnosed cancer in 28 countries and the leading cause of cancer death in 42 countries. [1]

More than $80 \%$ of carcinoma of the uterine cervix cases occur in developing countries with 4,83,000 estimated new cases and 2,74,000 deaths yearly. [2] In developing countries it is most commonly occurring genital cancer. This condition also seen in younger women with the average onset of age is 45 years. Life time risk of the carcinoma of uterine cervix is $2.4 \%$ in India. [3] The incidence peaks in middle age and ranges from 10 to 100 new cases/100000 population/year worldwide, based on the socioeconomic status of the different countries. [4]

Virtually all carcinoma of the uterine cervix is associated with Human Papilloma Virus (HPV) infection. However, the majority of woman do not develop cervical cancer following HPV infection but several other environmental factors are required for the development of cancer. [1] Cervical cancer is the commonest gynecologic malignancy in women younger than 50 years of age and from developing countries, particularly in those with a high prevalence of human papilloma virus (HPV) and human immunodeficiency virus (HIV). [5]

Cervical carcinoma mostly develops at the level of the squamocolumnar epithelial junction of the cervical mucosa. About $20 \%$ of tumours lie totally within the endocervical canal poses difficulties to be examined clinically. [4]

Preoperative clinical and image based staging is very important for management of patients with invasive cervical carcinoma, among the variables of staging size of tumour is very important for surgical planning. [6]

MR imaging examination obviates the use of invasive diagnostic procedures such as cystoscopy and proctoscopy, especially in cases of no sign of local extension. The best management option for each patient (i.e. surgery or radiation therapy) can be determined more accurately with the MR imaging, which has shown to influence treatment planning in half of patients. Brachytherapy and external beam therapy can be optimized kwith MR imaging evaluation of the shape and direction of growth. MR imaging can also be used in identification of important prognostic factors such as lesion volume and metastatic lymph node involvement that will help to determine whether the treatment would be palliative or curative. Surgery is the treatment modality of choice for stages at and lowers than IIA, except for lesions over $4 \mathrm{~cm}$ in diameter (stage IB2 or IIA). Radiation therapy alone or in combination with chemotherapy is preferred for stages IIB or higher or for lesions greater than $4 \mathrm{~cm}$. [15]

Cervical carcinoma exhibits intermediate signal intensity at T2-weighted imaging and is seen disrupting the lowsignal-intensity fibrous stroma. The tumor exhibits a wide variety of morphologic features and may be exophytic, infiltrating or endocervical with a barrel shape. In young women cervical carcinoma usually originates from the squamocolumnar junction and tends to be more exophytic, whereas in older women it originates more often in the endocervical canal. [15]

The bulk of the mass is centered at the level of the cervix, with either extension towards the vagina or to the lower myometrium of corpus. This permits differentiation from an endometrial mass (polyp or adenocarcinoma), which is centered in the endometrial cavity and protruding into the endocervical canal. Prolapsed submucous fibroids are hypointense at T2-weighted imaging. In general, carcinoma of the uterine cervix is better defined at T2-weighted images, but small tumors may be more readily identified by their early enhancement characteristics in dynamic images after injection of gadopentetate dimeglumine (Magnevist ${ }^{\mathrm{TM}}$ ). [15]

A visible tumor indicates stage IB or higher. The size of the tumor (whether greater or less than $4 \mathrm{~cm}$ in diameter) has a great impact on the choice of therapy, and there is good correlation between MR imaging findings and macroscopic measurements. However, the size of the lesion may rarely be overestimated at T2WI due to inflammation and edema. The shape and direction of growth should be noted because they are important for Brachytherapy planning. [15]

The management of cervical cancer particularly the surgical treatment, differs largely depending on the stage of the disease. Early cervical neoplasm can be cured with surgery. However, post- operative adjuvant therapy should be considered if pathologic findings demonstrate risk factors including lymph node metastases, deep cervical invasion, positive cut margin, parametrial invasion and bulkiness of tumour. Among them parametrial invasion and tumour size are evaluated by preoperative clinical examination. [6]

The most important issue in staging of cervical cancer is to distinguish early disease that can be treated with surgery from the advanced disease that must be managed with radiotherapy alone or in combination with chemotherapy. MRI is the single best modality that can accurately determine tumor location, tumor size, depth of stromal invasion and extension into the lower uterine segment. MRI is accurate for evaluation of tumor size usually with $0.5 \mathrm{~cm}$ difference of the surgical size in $90 \%$ cases. An important pitfall of MRI staging is that of over estimation of parametrial invasion on T2 weighted images in large tumors and accuracy of $70 \%$ compared with small one's accuracy of $96 \%$ due to stromal edema induced by tumor compression or inflammation. This may lead to higher rate of false positive assessment of parametrial invasion in patients with large tumors, which must be considered when making the decision of treatment of these patients. [3]

The objective of this study was to determine whether imaging modality could yield reliable results as a tool compared to pelvic examination for determination of actual tumour size in cervical cancer.

\section{Materials and Methods}

This cross-sectional analytical study was carried out in the Department of Radiology and Imaging, BSMMU, Dhaka in collaboration with the Department of Gynaecologic Oncology, between October 2018 to September 2020. Newly diagnosed biopsy proven cases of carcinoma cervix attended in the Department of Gynaecologic Oncology for clinical FIGO 
staging and then were referred to the Department of Radiology \& Imaging, BSMMU to perform MRI for image based staging fulfilling the inclusion \& exclusion criteria of diseased and were willing to participate in the study were taken as study population and the total number of patients were 60 .

Inclusion criteria:

1) Newly diagnosed cases of carcinoma cervix referred to the Department of Radiology and Imaging of BSMMU.

2) Purposive selection of the operable cases.

Exclusion criteria:

1) Previously treated cases of carcinoma cervix.

2) Pregnancy with carcinoma cervix.

3) Inoperable cases of carcinoma cervix.

\section{Results}

This cross-sectional study was carried out with an aim to evaluate the accuracy of MRI in determination of actual tumour size in carcinoma of uterine cervix in comparison to pelvic examination and to compare the findings with histopathology.

Table 1 demonstrates the characteristics of the patients. Most of the patients belonged to stage IIA, however, 6 women had findings consistent with stage IIB. Since the patients with advanced cervical cancer (stage IIB) were less than 50 years of age, the clinicians performed surgical treatment combined with postoperative adjuvant therapy for these patients. Tumor size was $4.44 \pm 1.28 \mathrm{~cm}$. (mean of the maximum diameter \pm standard deviation), and ranged from 1.5 $\mathrm{cm}$ to $7.0 \mathrm{~cm}$ at pathologic evaluation. Histopathologic examination of the majority of patients revealed squamous cell carcinoma.

Table 1. Characteristics of patients.

\begin{tabular}{ll}
\hline Number & \\
\hline Mean age (year) & $47.5 \pm 10.1$ \\
FIGO stage & 20 \\
IB & 34 \\
IIA & 06 \\
IIB & \\
Histology & 42 \\
Squamous cell carcinoma & 14 \\
Adenocarcinoma & 04 \\
Adeno-squamous & $4.44 \pm 1.28 \mathrm{~cm}$. \\
Mean tumour size & \\
\hline
\end{tabular}

Table 2 depicts the accuracy of pelvic examination and imaging modality in the measurement of tumor size compared with the "true" value. Based on pathologic findings, accuracy was estimated by the degree of agreement with a difference of 0.5 or $1.0 \mathrm{~cm}$. Imaging modality was more accurate in the evaluation of tumor size than pelvic examination with a difference of both $<0.5$ and $0.5-1.0 \mathrm{~cm}$, and the accuracy rate of imaging modality was significantly higher than that of pelvic examination $(75 \%$ vs.53.3\%) in 0 . $<5 \mathrm{~cm}$ with $100 \%$ vs $88.3 \%$ in $0.5 \mathrm{~cm}-1.0 \mathrm{~cm}$ range.
Table 2. Accuracy of pelvic examination and imaging modality for the measurement of tumor diameter.

\begin{tabular}{lll}
\hline Accuracy $(<\mathbf{0 . 5} \mathbf{~ c m})$ & & Accuracy $(\mathbf{0 . 5}-\mathbf{1 . 0} \mathbf{c m})$ \\
\hline Pelvic examination & $53.3 \%$ & $75 \%$ \\
Imaging mk2odality (MRI) & $88.3 \%$ & $100 \%$ \\
\hline
\end{tabular}

Following Table 3 showing that majority of the detected tumor size were $\geq 4 \mathrm{~cm}$ in all three modalities, among them 39 cases were found in histopathology, 41 cases were found in MRI and 36 were found in clinical FIGO staging.

Table 3. Distribution of the study patients according to tumor size $(n=60)$.

\begin{tabular}{lll}
\hline Tumor size & Number of patients & Percentage (\%) \\
\hline MRI & & \\
$1.5-2.0 \mathrm{cmk}$ & 01 & 1.67 \\
$2.0-3.9 \mathrm{~cm}$ & 18 & 30.00 \\
$4.0-7.0 \mathrm{~cm}$ & 41 & 68.33 \\
Clinical FIGO & & \\
$1.5-2.0 \mathrm{~cm}$ & 00 & 0.0 \\
$2.0-3.9 \mathrm{~cm}$ & 24 & 40.0 \\
$4.0-7.0 \mathrm{~cm}$ & 36 & 60.0 \\
Histopathology & & \\
$1.5-2.0 \mathrm{~cm}$ & 01 & 1.7 \\
$2.0-3.9 \mathrm{~cm}$ & 20 & 33.3 \\
$4.0-7.0 \mathrm{~cm}$ & 39 & 65.0 \\
\hline
\end{tabular}

Table 4 shows that 1 patient was found tumor differed $\leq 0.5$ $\mathrm{cm}$ from histopathological tumor size in $<2.0$ category in FIGO, 9 patient differed in $2.0-3.9 \mathrm{~cm}$ category and 18 patients in $\geq 4.0 \mathrm{~cm}$ category of actual tumour size. In MRI, 1 patient was found tumor differed $\leq 0.5 \mathrm{~cm}$ from histopathological tumor size in $<2.0 \mathrm{~cm}$ category, 3 in 2.0-3.9 $\mathrm{cm}$ and 11 in $\geq 4.0 \mathrm{~cm}$ category of actual tumor size. In case of tumour size differed $>0.5-1.0 \mathrm{~cm}$, clinical staging showing 7 cases differed in $\geq 4.0 \mathrm{~cm}$ category of actual tumour size whether MRI not differed any case in this category.

Table 4. Comparison of differences of pelvic examination and MRI size of tumor based on histopathological tumor size $(n=60)$.

\begin{tabular}{lccl}
\hline \multirow{2}{*}{ Tumor size } & \multicolumn{3}{l}{ Histopathological tumor size } \\
\cline { 2 - 4 } & $<\mathbf{2 . 0} \mathbf{~ c m}$ & $\mathbf{2 . 0 - 3 . 9} \mathbf{~ c m}$ & $\mathbf{2 4 . 0} \mathbf{~ c m}$ \\
\hline \multicolumn{4}{l}{ Accuracy with a difference of $\leq 0.5 \mathrm{~cm}$} \\
Pelvic examination & $1(1.7 \%)$ & $9(15.0 \%)$ & $18(30.0 \%)$ \\
MRI & $1(1.7 \%)$ & $3(5.0 \%)$ & $11(18.3 \%)$ \\
Accuracy with a difference of $>0.5-1.0 \mathrm{~cm}$ & \\
Pelvic examination & $0(0.0 \%)$ & $0(0.0 \%)$ & $7(11.7 \%)$ \\
MRI & $0(0.0 \%)$ & $0(0.0 \%)$ & $0(0.0 \%)$ \\
\hline
\end{tabular}

Figure 1 scatter diagram showing the predictability of the relationship of tumour size obtained in MRI where the histopathologic tumour size (independent variable) is plotted in ' $\mathrm{X}$ ' axis and MRI tumour size (dependent variable) is plotted in ' $\mathrm{Y}$ ' axis. It showing strong linear positive correlation of the MRI tumour size as most of the values are closely approximated with the line of best fit.

Figure 2 scatter diagram showing the predictability of the relationship of tumour size obtained in clinical FIGO where the histopathologic tumour size (independent variable) is plotted in ' $\mathrm{X}$ ' axis and FIGO tumour size (dependent variable) is plotted in ' $Y$ ' axis. It showing moderate linear positive correlation of the FIGO tumour size as most of the values are approximated with the line of best fit. 


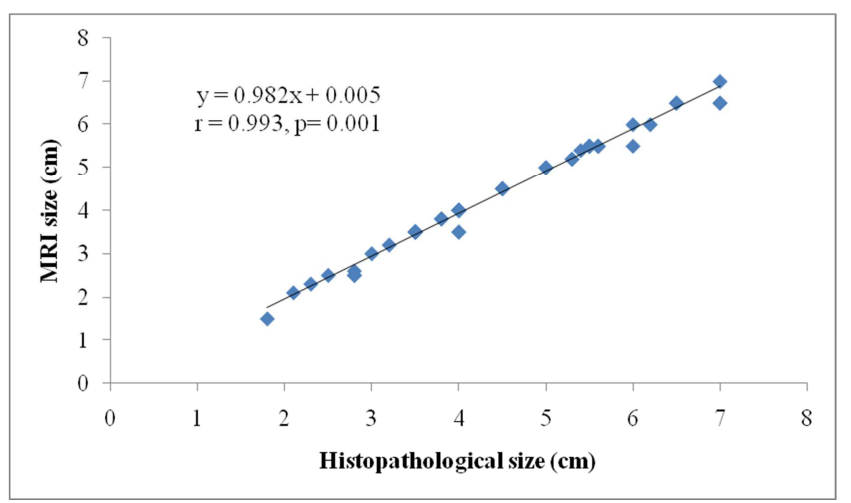

Figure 1. Scatter diagram illustrates positive correlation $(r=0.993 ; p=0.001)$ between histopathological and MRI tumour size.

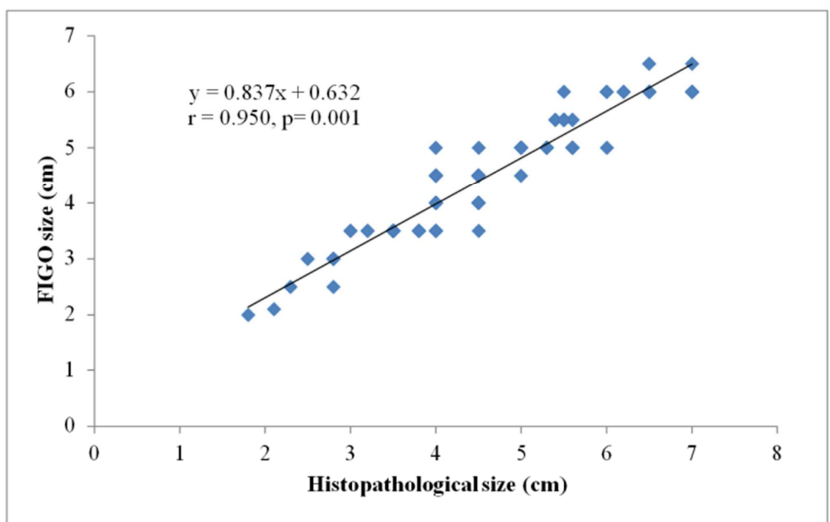

Figure 2. Scatter diagram illustrates positive correlation $(r=0.950 ; p=0.001)$ between histopathological and clinical tumour size.

\section{Discussion}

Apart from gynecologic malignancies i.e. ovarian cancer and endometrial carcinomas whose stages are determined surgically, the stage of cervical cancer is evaluated clinically. Thus, there are several prognostic factors which are not determinants of cervical cancer staging. Since some pathologic parameters are related to patients' prognosis, these parameters affect the plan of further treatment. [7-10] This means that pathologic findings of surgical specimens are important for the management of patients with cervical cancer. Clinicians can get much information related to prognosis from preoperative clinical diagnosis and staging. Tumor size been suggested as an important prognostic factor in patients with cervical cancer. [11-14]

Although there are several other diagnostic methods including pelvic examination, IVP, cystoscopy, proctoscopy and many others, pelvic examination is the only single best and accepted method for evaluating the size, location and parametrial invasion of the cervical mass. Thus, exact pelvic examination is crucial for the management of patients with carcinoma cervix. Recent development of imaging techniques and modalities have made it possible to evaluate not only the characteristics of cervical mass but also the status of lymph nodes. MRI has progressively replaced standard diagnostic methods of invasive cervical neoplasm. Moreover, some clinicians make a decision for the management of cervical cancer using imaging tools such as CT or MRI instead of the pelvic examination.

The current study demonstrates the accuracy of pelvic examination and the imaging modality for the evaluation of cervical mass in 60 cervical cancer patients. The real size of cervical mass ranged from 1.5 to $7.0 \mathrm{~cm}$ in pathologic findings. Compared with the size of the cervical mass evaluated by pathologic findings, MRI estimated the size of the cervical mass more accurately than pelvic examination. Many previous studies focused on the value of imaging modalities as new diagnostic method for evaluating tumor size, parametrial invasion and lymph node involvement.

In this study we observed that majority $(50.0 \%)$ of the patients belonged to age group of 51-60 years. The mean age was found $47.5 \pm 10.1$ years, ranging from 22 to 60 years.

In this study observed that $37(61.7 \%)$ patients were found tumor size $\geq 4 \mathrm{~cm}$ in MRI, $36(60.0 \%)$ in FIGO and $39(65.0 \%)$ in histopathological findings. In present study the mean tumour size were found in MRI $4.37 \pm 1.26 \mathrm{~cm}$, FIGO $4.35 \pm 1.13 \mathrm{~cm}$, histopathology $4.44 \pm 1.28 \mathrm{~cm}$. Lee et al.,(2008) observed that the mean tumor size was $2.76 \pm 1.76 \mathrm{~cm}$ with ranged from $0.5 \mathrm{~cm}$ to $8.0 \mathrm{~cm}$ at pathologic evaluation. The mean tumor size is differed in our study due to presence of more number of cases in stage IIA1 and IIA2.

Although quite a few studies have reported the value of pelvic examination in cervical cancer, it has been demonstrated that the accuracy of pelvic examination is $53.3 \%$ with a difference of $<0.5 \mathrm{~cm}$ from the actual values and $75 \%$ in MRI. In the category of $0.5 \mathrm{~cm}-1.0 \mathrm{~cm}$ of tumour size difference the accuracy of pelvic examination findings is about $88.3 \%$ and is almost $10 \%$ in case of MRI. These results were not similar to findings of the former previous studies and the increasing accuracy of the imaging modality due to improvement of the facility and the quality of Radiologists.

In present study observed positive correlation $(\mathrm{r}=0.993$; $\mathrm{p}=0.001$ ) between histopathological size and MRI size. Positive correlation $(\mathrm{r}=0.950 ; \mathrm{p}=0.001)$ between histopathological size and FIGO size. In this study MRI findings more correlated with histopathology than clinical FIGO staging in detecting tumor size. Lee et al., (2008) reported that the correlation with pathologic findings was higher for pelvic examination than for imaging modality ( $r s=0.680$ vs. $r s=0.410, \mathrm{p}<0.001)$. Our study was more in favor of MRI due to improvement of imaging parameters and radiologist interpretation.

Finally, the study observed histopathology and MRI staging concurred in $95.0 \%$ cases and differed in $5.0 \%$ cases. FIGO staging concurred with Histopathology in $65.0 \%$ cases and differed in $35.0 \%$ cases. Lee et al. (2008) reported some investigators have found the accuracy of MRI ranged about from $30 \%$ to $70 \%$ according to the tumor size. Some reports have demonstrated the accuracy of MRI in the evaluation of the stage of cervical cancer ranged from $70 \%$ to $85 \%$.

\section{Conclusions}

This study was undertaken to evaluate the sensitivity and 
specificity of MRI in detection of tumour size as a part of staging of carcinoma cervix in comparison to the pelvic examination taking histopathology as gold standard. The MRI size of invasive carcinoma cervix was significantly well correlated with histopathology, as the validity test results were higher. So in the light of the findings of present study it can be concluded that MRI is a useful noninvasive imaging modality in preoperative staging of cervical carcinoma and determination of actual tumour size as well.

\section{Recommendations}

Biological prognostic factors (e.g. histologic grades, Lymph-vascular space invasion, serum biomarkers, etc) be included in current staging system, with an aim of better identification of patients at high and low risk of dying of their disease. Further works needed to assure the consistency and uniformity among pathologists when rendering tumor size measurements in patients with early cervical cancer. Financial aspects also need to be considered in using MRI in place of conventional clinical staging tests according to clinical indication.

\section{References}

[1] Bray, F., Ferlay, J., Soerjomataram, I., Siegel, R. L., Torre, L. A. and Jemal, A., 2018. Global cancer statistics 2018: GLOBOCAN estimates of incidence and mortality worldwide for 36 cancers in 185 countries. CA: a cancer journal for clinicians, 68 (6), pp. 394-424.

[2] Stenstedt K, Hellström AC, Fridsten S, Blomqvist L. Impact of MRI in the management and staging of cancer of the uterine cervix. Acta oncologica. 2011 Apr 1; 50 (3): 420-6.

[3] Kumari, D. A. and Paga, A., 2017. "Comparative study of MRI staging Vs FIGO staging of carcinoma cervix." International journal of contemporary medical research, 4 (5), pp. 11961198.

[4] Kirova G, Nedialkov K, Sergieva S. Magnetic resonance imaging and its value in the staging of cervical carcinomaComparison of magnetic resonance imaging and pathological images with FIGO staging system. JOURNAL-BALKAN UNION OF ONCOLOGY. 2004 Apr 1; 9: 127-38.

[5] Sauer J, Simonds HM, Van der Merwe H, Hattingh SM. A retrospective analysis comparing clinical staging with magnetic resonance imaging staging in patients with cervical cancer. Southern African Journal of Gynaecological Oncology. 2013 Jan 1; 5 (1): 11-5.

[6] Lee YK, Han SS, Kim JW, Park NH, Song YS, Kang SB. Value of pelvic examination and imaging modality for the evaluation of tumor size in cervical cancer. Journal of gynecologic oncology. 2008 Jun; 19 (2): 108.

[7] Zaino RJ, Ward S, Delgado G, Bundy B, Gore H, Fetter G, Ganjei P, Frauenhofer E. Histopathologic predictors of the behavior of surgically treated stage IB squamous cell carcinoma of the cervix a gynecologic oncology group study. Cancer. 1992 Apr 1; 69 (7): 1750-8.

[8] Vinh-Hung V, Bourgain C, Vlastos G, Cserni G, De Ridder M, Storme G, Vlastos AT. Prognostic value of histopathology and trends in cervical cancer: a SEER population study. BMC cancer. 2007 Dec; 7 (1): 1-3.

[9] Metindir J, Bilir G. Prognostic factors affecting disease-free survival in early-stage cervical cancer patients undergoing radical hysterectomy and pelvic-paraaortic lymphadenectomy. European journal of gynaecological oncology. 2007 Jan 1; 28 (1): 28-32.

[10] Chung CK, Nahhas WA, Stryker JA, Curry SL, Abt AB, Mortel R. Analysis of factors contributing to treatment failures in stages IB and IIA carcinoma of the cervix. American Journal of Obstetrics and Gynecology. 1980 Nov 1; 138 (5): 550-6.

[11] Homesley HD, Raben M, Blake DD, Ferree CR, Bullock MS, Linton EB, Greiss Jr FC, Rhyne AL. Relationship of lesion size to survival in patients with stage IB squamous cell carcinoma of the cervix uteri treated by radiation therapy. Surgery, gynecology \& obstetrics. 1980 Apr 1; 150 (4): 529-31.

[12] Kristensen GB, Abeler VM, Risberg B, Tropé C, Bryne M. Tumor size, depth of invasion, and grading of the invasive tumor front are the main prognostic factors in early squamous cell cervical carcinoma. Gynecologic oncology. 1999 Aug 1; 74 (2): 245-51.

[13] Trattner M, Graf AH, Lax S, Forstner R, Dandachi N, Haas J, Pickel H, Reich O, Staudach A, Winter R. Prognostic factors in surgically treated stage $\mathrm{Ib}-\mathrm{IIb}$ cervical carcinomas with special emphasis on the importance of tumor volume. Gynecologic oncology. 2001 Jul 1; 82 (1): 11-6.

[14] Horn LC, Fischer U, Raptis G, Bilek K, Hentschel B. Tumor size is of prognostic value in surgically treated FIGO stage II cervical cancer. Gynecologic oncology. 2007 Nov 1; 107 (2): 310-5.

[15] Nicolet V, Carignan L, Bourdon F, Prosmanne O. MR imaging of cervical carcinoma: a practical staging approach. Radiographics. 2000 Nov; 20 (6): 1539-49. 